CERN-TH.6595/92

ACT-17/92

CTP-TAMU-58/92

\title{
String Theory Modifies Quantum Mechanics
}

\author{
John Ellis, N.E. Mavromatos and D.V. Nanopoulos ${ }^{\dagger}$ \\ Theory Division, CERN, CH-1211, Geneva 23, Switzerland
}

\begin{abstract}
We argue that the light particles in string theory obey an effective quantum mechanics modified by the inclusion of a quantum-gravitational friction term, induced by unavoidable couplings to unobserved massive string states in the space-time foam. This term is related to the $W$-symmetries that couple light particles to massive solitonic string states in black hole backgrounds, and has a formal similarity to simple models of environmental quantum friction. It increases apparent entropy, and may induce the wave functions of macroscopic systems to collapse.
\end{abstract}

CERN-TH.6595/92

ACT-17/92

CTP-TAMU-58/92

July 1992

$\dagger$ Permanent address: Center for Theoretical Physics, Dept. of Physics, Texas A \& M University, College Station, TX 77843-4242, USA, and

Astroparticle Physics Group, Houston Advanced Research Center (HARC), The Woodlands, TX 77381, USA. 


\section{Introduction}

The discoveries of quantum mechanics and general relativity have caused two of the greatest revolutions in twentieth-century physics, and their reconciliation remains one of its most important pieces of unfinished business. One could expect that this reconciliation would entail a modification of one or both of these very successful theories, and hope that it would cast light on the transition between classical and quantum physics. Indeed, the only candidate for a consistent quantum theory of gravity is string theory [1], which is essentially non-local and hence modifies general relativity at short distances. It has also been suggested that the usual formulation of quantum mechanics and quantum field theory might require modifications in a consistent quantum theory of gravity. Specifically, studies of field theory in topologically non-trivial space-times such as black hole backgrounds have indicated that information loss across an event horizon requires the introduction of mixed quantum-mechanical states, and the possibility, forbidden by conventional quantum mechanics or $S$-matrix theory, that pure states may evolve into mixed states.

It has been proposed by Hawking [2] that a full quantum theory of gravity might be formulated only in terms of density matrices describing in general mixed states, and that the transitions between initial and final density matrices might not be factorizable as products of $S$-matrix elements and their hermitian conjugates:

$$
\rho_{\text {out }}=\not \rho_{\text {in }} \quad: \quad \$ \neq S^{\dagger} S
$$

Evidence for this suggestion was inferred from studies of topologically non-trivial solutions of Einstein's equations in Euclidean space-times. Two of us (J.E. and D.V.N.) then suggested together with J.S. Hagelin and M. Srednicki [3] that the evolution of quantum-mechanical systems over time-scales that are long compared with the Planck time should be described by a modified Liouville equation:

$$
\partial_{t} \rho=i[\rho, H]+\phi H \rho
$$

A modification of the Liouville equation of the type (2) is characteristic of open quantum-mechanical systems [由], and represents in our interpretation the intrinsic coupling of a microscopic system to space-time foam.

We derived upper bounds of the order of $1 G e V / M_{\text {Planck }}$ on the magnitude of matrix elements of the $\phi H$ term in hadrons from the consistency with conventional quantum mechanics of measurements of the $K^{0}-\bar{K}^{0}$ system [3, 5] and long-baseline neutron interferometry [3]. Subsequently, the same two of us together with S. Mohanty [6] demonstrated that the non-quantum-mechanical effects of the $\phi H$ would be enhanced in macroscopic systems such as SQUIDs, and could lead to the collapse of the wave function of a macroscopic object. An operationally similar modification of the Liouville equation was proposed independently on completely phenomenological grounds by Ghirardi, Rimini and Weber [7], and the required values of their model 
parameters were entirely consistent with our upper bounds and order-of-magnitude estimates.

The study of topologically non-trivial space-times, event horizons and singularities in string theory was opened up by Witten's realization that suitable Wick rotations of a cosmological string theory could be interpreted as black holes in Minkowski or Euclidean space. We then embarked on a series of studies of quantum coherence in such stringy black hole backgrounds, with the explicit motivation of checking the conjectured breakdowns (11) and (2) of conventional quantum field theory and quantum mechanics. We found that quantum coherence was maintained in the scattering of light particles on a black hole background by an infinite set of local $W_{1+\infty}$-symmetries [8, 9], which link light asymptotic states to massive string states, and whose associated conserved $W$-charges could encode all initial-state information. We believe that quantum coherence can be maintained in this situation only in an intrinsically non-local theory such as string with its infinite set of gauge symmetries, and that local field theories are doomed to failure in this respect because they only have a finite set of hair. We have shown that the infinite set of stringy $W$-hair is sufficient to label all the black hole states, and thereby quench all the entropy $S=M^{2}$ of a spherically-symmetric black hole in four dimensions 110 . We have also argued [11] that black hole decay is a purely quantum-mechanical higher-genus effect that does not require a thermal description in terms of Hawking radiation, and that all the $W$-charges are in principle measurable via scattering experiments or Aharonov-Bohm phases for massive string states [12]. We have also given a stringy desription of space-time foam in terms of a plasma of defects on the world-sheet, which is intimately related to a Hall conductor [13].

The maintenance of quantum coherence in the scattering of light particles off a stringy black hole does not, however, mean that conventional quantum mechanics and quantum field theory are sacrosanct. The essential problem is that although all the $W$-charges are in principle measurable, this would entail observations of the massive string states that are linked to the light states by the $W$-symmetries, and such observations are not in practice possible in realistic laboratory experiments. We argue in this paper that a modification of the Liouville equation of the type (2) is indeed essential for observable systems containing only light particles. Its origin is exactly the $W$-linkage between the light and heavy string states, which means that the former must be regarded as an open system coupled to the unobserved heavy degrees of freedom. A modification of the form (2) would be forbidden for any exactly marginal deformation of the underlying string theory. However, it is known from explicit examples that in non-trivial space-time backgrounds such exactly marginal deformations involve massive string states. Therefore, any deformation of the effective theory of light particles in which the massive modes are unobserved will necessarily not be exactly marginal, and will hence be associated with a contribution to $\phi H$ (2). The unitarity of the effective field theory of the light degrees of freedom and Zamolodchikov's c-theorem [14], as proved by one of the authors 
(N.E.M.) and Miramontes [15], guarantee that any contribution to $\phi H$ can only increase the entropy of the light-particle system. Topologically non-trivial space-time backgrounds appear intrinsically in the stringy realization of space-time foam, so we conclude that a $\phi H$ term is inevitable in string theory. We argue, moreover, that the dominant such modifications are just those associated with the known $S$-wave deformations of spherically-symmetric black holes. A primitive order-of-magnitude estimate indicates that their contribution to $\phi H$ might be suppressed by just one inverse power of $M_{\text {Planck }}$, and hence be close to the present experimental upper limits [3], 5], and in the ball-park needed to explain the collapse of the wave function for a classical system.

\section{Renormalization Group Flow as Quantum Fric- tion}

Let us consider a general dynamical system whose state is described by a density matrix $\rho\left\{q^{i}, p_{i}\right\}$, where the $q^{i}$ are generalized coordinates, and the $p_{i}$ are their associated conjugate momenta. In conventional quantum mechanics, the $q^{i}$ include conventional space-time coordinates, which in string theory become parameters of background target spaces corresponding to $\sigma$-models on the world-sheet. Thus the $q^{i}$ can be regarded as couplings in a space of possible $\sigma$-models. We consider the evolution of $\rho$ with respect to a renormalization group flow variable $t=\ln \Lambda$, where $\Lambda$ is some covariant cutoff. We will identify $t$ with a conventional time variable, which may or may not be considered as a string Liouville mode. On dimensional grounds, the target time is then measured in units of Planck length. This in turn implies that even marginal changes in the renormalisation group scale produce appreciable time-variations in target-space. It is clear that renormalisability of the system implies

$$
\frac{d \rho}{d t}=0=\frac{\partial \rho}{\partial q^{i}} \dot{q}^{i}+\frac{\partial \rho}{\partial p_{i}} \dot{p}_{i}
$$

where $(. .$.$) denotes \partial_{t}(\ldots)$.

It is known [16] that in the Wilson renormalization scheme the renormalization flow of the coordinates ( $\sigma$-model couplings) $q^{i}$ is given in the neighbourhood of a fixed point $S_{0}$ by the gradient of Zamolodchikov's $c$-function $\Phi\left(q^{i}, S_{0}\right)$ :

$$
\beta^{i} \equiv \dot{q}^{i}=G_{i j}\left(S_{0}\right) \frac{\partial \Phi\left(q^{i}, S_{0}\right)}{\partial q^{i}}
$$

where $G_{i j}$ is the metric in the space of coupling constants:

$$
G_{i j} \equiv 2|z|^{2}<O_{i}(z, \bar{z}) O_{j}(0,0)>
$$

with $<\ldots>$ denoting an average with respect to the deformed $\sigma$-model action $I^{*}+\int d^{2} z q^{i} O_{i}$, where $I^{*}$ is the fixed-point conformal field theory action, and the $O_{i}$ are a complete set of (renormalised) vertex operators. 
In view of equation (4), we can regard $\Phi$ as a Lagrange function, to which we can associate a Hamiltonian in the usual way:

$$
\Phi=\int d t\left[\dot{q}^{i} \cdot p_{i}-H\left(q^{i}, p_{i}\right)\right]
$$

Again in the usual way, it follows that

$$
\dot{q}^{i}=\frac{\partial H}{\partial p_{i}}
$$

and

$$
\dot{p}_{i}=-\frac{\partial H}{\partial q^{i}}-G_{i j} \beta^{j}
$$

The second term in equation (8) is a friction term characteristic of open dynamical systems [4, which will be at the root of our subsequent modification of quantum mechanics 1. Substituting the expressions (7), (8) into the time derivative equation (3), we find the following equation

$$
\dot{\rho}=i[\rho, H]+G_{i j} \beta^{j} \frac{\partial \rho}{\partial p_{i}}
$$

for the classical evolution of the density matrix 2. When proposing any such modification of the traditional evolution equation for the density matrix, it is important to check that the total probability $P=\int d p_{i} d q^{i} \operatorname{tr} \rho\left(p_{i}, q^{i}\right)$ is conserved. In our case (9), it is easy to check that

$$
\partial_{t} P=\int d p_{l} d q^{l} \operatorname{tr} \frac{\partial}{\partial p_{i}}\left(G_{i j}(q) \beta^{j}(q) \rho(p, q)\right)
$$

which vanishes if there are no contributions from the boundary of phase space, as would occur if it has no boundaries, or if $\rho$ vanishes there.

The quantum version of this classical equation is obtained by first replacing

$$
\{,\} \rightarrow \frac{1}{i}[,]
$$

\footnotetext{
${ }^{1}$ In the Wilson renormalisation scheme (4) the friction is linear, which simplifies many of the computations. However in practice, or in certain cases where this scheme is not applicable [16], one can work in schemes where (1) is valid but $G_{i j}=G_{i j}(q)$, in which case one is faced with a non-linear friction problem. For most of our discussion we can stay in the general case.

${ }^{2} \mathrm{~A}$ similar equation has been considered by Kogan [17], but his formalism is incorrect because he considers total time derivatives in places where one should consider partial ones. In addition, he identifies the Liouville field with the target time coordinate, which clearly does not apply to the two-dimensional black hole string theory [18, 19]. We stress again that from our point of view the world-sheet cutoff is identified with the target time (evolution parameter) independently of any (possible) identification of the Liouville field with time.
} 
The quantum version of the second term in (9) is obtained by recalling Euler's equation (7), whose quantum version is

$$
\frac{\partial H}{\partial p_{i}}=-i\left[q^{i}, H\right]
$$

from which it follows that

$$
\frac{\partial F(H)}{\partial p_{i}}=-i\left[q^{i}, F(H)\right]
$$

for any function $F$ of the Hamiltonian, including in particular the density matrix $\rho$. We therefore arrive at the folowing evolution equation for the quantum density matrix:

$$
\dot{\rho}=i[\rho, H]-i G_{i j}\left[\rho, q^{i}\right] \beta^{j}
$$

We will not enter here into a discussion of the appropriate quantum ordering of the factors in the second term in equation (14), which represents the quantum friction inherent to our open dynamical system.

An additional remark we would like to make concerns the connection of the gradient flow relation (14) with the problem of a supersymmetric quantum mechanical particle moving in a constant magnetic field ( more specifically in a Kahler potential $\Phi(q)$ [20]). Indeed, it can be shown [20] that whenever relation (4) is valid, the evolution of $q^{i}$ in $t=\ln \Lambda$ can be described by a super-quantum mechanics Hamiltonian $H=\frac{1}{2}\left(Q+Q^{\dagger}\right)$, with supercharge $Q=\psi^{i}\left(\nabla_{i}-\beta_{i}\right)$ and its hermitian conjugate $Q^{\dagger}$, where the $\psi^{i}$ are Grassmann variables satisfying canonical commutation relations. The charges are nilpotent : $Q^{2}=\left(Q^{\dagger}\right)^{2}=0$ if and only if the covariant $\beta_{i}$ are curl-free, i.e. if relation (便) is satisfied. In this case the metric in coupling constant space can be viewed as a Kahler metric, whose potential coincides with the flowfunction $\Phi(q)[20]$. This analogy makes clearer the physical interpretation of the renormalisation group flow as a friction problem related to the quantum motion of a particle in a magnetic field [21].

Our previous demonstration that the total probability $P$ is conserved carries over directly from the classical case (9) to the quantum case (14). However, entropy is $S=-\operatorname{tr} \rho \ln \rho$ is not necessarily conserved: we see easily that

$$
\dot{S}=-\operatorname{tr} G i j\left[\rho, q^{i}\right] \beta^{j} \ln \rho
$$

which does not in general vanish if $\beta^{i} \neq 0$. Our next task is to demonstrate that indeed $\beta_{i} \equiv G_{i j} \beta^{j} \neq 0$, and in so doing we will be able to argue that the entropy $S$ increases monotonically, as one would expect on general physical grounds. 


\section{Light Particle Operators are not Exactly Marginal}

Since string theory is based on conformal field theory, one might naively expect that the renormalization coefficients $\beta^{i}$ introduced above all vanish, and hence that the quantum friction term in equation (14) vanishes identically. This is indeed the case in a flat space-time background, but is not true for operators that create light particles in non-trivial backgrounds. The prototype for this phenomenon is the operator creating a massless particle in a two-dimensional string black hole background, which can also be interpreted as a spherically-symmetric four-dimensional black hole background. We first present a heuristic argument that $\beta^{i} \neq 0$ in this case, which is followed by a more complete treatment in section 5 .

We recall that the black hole solution possesses a $W_{1+\infty}$-symmetry that is global on the world-sheet, and includes the Virasoro algebra. It becomes a local symmetry when elevated in target space, providing an infinite-dimensional extension of the general coordinate transformations of general relativity. This $W$-symmetry is responsible, in our interpretation, for the existence of the $S$-matrix for scattering massless particles, confusingly called "tachyons", in this black hole background, and hence for the maintenance of quantum coherence in this scattering process. The feature of these $W$-symmetries that is crucial for the present discussion is that they link together states with different masses. In particular, they relate massless "tachyon" states to massive string levels. One could therefore suspect that the conformal subalgebra of the $W$-symmetry associated with the Virasoro algebra might also combine massless and massive states in an essential way, and we shall see in a moment that such is indeed the case. Therefore, the $\beta$-functions for operators contructed out of light particle fields alone will not have the full $W$-symmetry, and hence not be conformal in general, and hence have some $\beta^{i} \neq 0$. But physical laboratory experiments are conducted with light particles, corresponding to states that are massless in the approximation which we discuss here, and do not measure any properties of the massive string states. Therefore the relevant vertex operators are precisely those that involve only massless states, and hence have non-vanishing $\beta$-functions. Hence the effective quantum mechanics of observable particles has the extra quantum friction term in (14), even though the density matrix of the full string theory would be described by the usual Liouville equation without such a term. The light observable particles constitute an open quantum-mechanical system coupled by $W$-symmetry to unobserved massive states.

Examples of the above assertion that in non-trivial backgrounds exactly marginal deformations generally involve massive string states have been provided in ref. [22]. In two-dimensional flat-space Liouville theory, the following "tachyon" vertex operator is exactly marginal:

$$
\phi_{-1 / 2,0,0}^{c,-c}=\left(g_{++} g_{--}\right)^{-\frac{1}{2}} F\left(\frac{1}{2} ; \frac{1}{2} ; 1 ; \frac{g_{+-} g_{-+}}{g_{++} g_{--}}\right)
$$


where $g_{a b}, a, b=+,-$ represent the components of a generic $S L(2, R)$ element [23]. However, the corresponding exactly marginal operator in a two-dimensional Minkowski space-time black hole, described by an $\operatorname{SL}(2, \mathrm{R}) / \mathrm{O}(1,1)$ Wess-Zumino (WZ) coset model, is

$$
L_{0}^{1} \bar{L}_{0}^{1}=\phi_{-1 / 2,0,0}^{c,-c}+i\left(\psi^{++}-\psi^{--}\right)+\ldots
$$

where

$$
\psi^{ \pm \pm} \equiv:\left(\bar{J}^{ \pm}\right)^{N}\left(J^{ \pm}\right)^{N}\left(g_{ \pm \pm}\right)^{j+m-N}:
$$

with $J^{ \pm} \equiv(k-2)\left(g_{ \pm \mp} \partial_{z} g_{ \pm \pm}-g_{ \pm \pm} \partial_{z} g_{ \pm \mp}\right)$, and $\bar{J}^{ \pm} \equiv(k-2)\left(g_{\mp \pm} \partial_{\bar{z}} g_{ \pm \pm}-g_{ \pm \pm} \partial_{\bar{z}} g_{\mp \pm}\right)$, with $k$ the WZ model level parameter [23]. The combination $\psi^{++}-\psi^{--}$generates a level-one massive string mode, and the dots in equation (17) represent operators that generate higher-level massive string states. An analogous exactly marginal opearator is

$$
L_{0}^{2} \bar{L}_{0}^{2}=\psi^{++}+\psi^{--}+\psi^{-+}+\psi^{+-}+\ldots
$$

which also involves in an essential way operators for massive string modes. For later use we note that the coupling constant, $\alpha$, corresponding to this deformation of the coset model is responsible for a global rescaling of the target space-metric [22], and therefore to a global constant shift by $\alpha$ of the dilaton field. Thus it produces shifts in the black hole mass 23].

We now remark that in two-dimensional string theory all these massive states are in fact discrete states of the type generated by the $W_{1+\infty}$ algebra which maintains quantum coherence [8, 24. Each of these discrete solitonic states can be represented as a singular gauge configuration [25], whose conserved $W$-charges can be measured in principle by generalized Aharonov-Bohm phase effects [12]. However, laboratory measurements of light microscopic objects such as the $K^{0}-\bar{K}^{0}$ system or interfering neutron beams do not measure such effects, so do not observe the massive states and are restricted to the light (massless) parts of the exactly marginal operators (17, 19). These by themselves are not exactly marginal, hence the corresponding light-field $\beta$ functions do not vanish, and therefore make non-zero contributions to the quantum friction term in equation (14). We shall give order of maginitude estimates of these effects in the next section.

As we have discussed in ref. 13 the sum over quantum configurations in the string path integral includes a sum over many vortices and spikes on the world-sheet:

$$
Z=\int D \tilde{X} \exp \left(-\beta S_{e f f}(\tilde{X})\right)
$$

where $\tilde{X} \equiv \beta^{\frac{1}{2}} X, \beta$ is a 'temperature' variable characterising the topological defects on the world-sheet, and

$$
\begin{aligned}
\beta S_{e f f} & =\int d^{2} z\left[2 \partial \tilde{X} \bar{\partial} \tilde{X}+\frac{1}{4 \pi}\left[\gamma_{v} \epsilon^{\frac{\alpha}{2}-2}(2 \sqrt{|g(z)|})^{1-\frac{\alpha}{4}}: \cos (\sqrt{2 \pi \alpha}[\tilde{X}(z)+\tilde{X}(\bar{z})]):\right.\right. \\
& \left.\left.+\left(\gamma_{v}, \alpha, \tilde{X}(z)+\tilde{X}(\bar{z})\right) \rightarrow\left(\gamma_{m}, \alpha^{\prime}, \tilde{X}(z)-\tilde{X}(\bar{z})\right)\right]\right]
\end{aligned}
$$


Here $\gamma_{v, m}$ are the fugacities for vortices and spikes respectively, and

$$
\alpha \equiv 2 \pi \beta q_{v}^{2} \quad \alpha^{\prime} \equiv \frac{e^{2}}{2 \pi \beta}
$$

are related to the conformal dimensions $\Delta_{v, m}$ of the vortex and spike creation operators respectively, namely

$$
\begin{array}{r}
\alpha=4 \Delta_{v} \quad \alpha^{\prime}=4 \Delta_{m} \\
\Delta_{m}=\frac{\left(e q_{v}\right)^{2}}{16 \Delta_{v}}
\end{array}
$$

In the low-temperature phase relevant in the present-day Universe, this path-integral is dominated by a plasma of world-sheet spikes corresponding to microscopic Minkowskispace black holes, which constitute a stringy realization of space-time foam 13. Therefore, many configurations with non-zero values of the $\beta^{i}$ contribute importantly to the string path integral, and one cannot a priori expect the modification (14) of the quantum Liouville equation to be negligible.

The reader might worry that although individual configurations contribute to the quantum fiction term in (14), some as yet unknown symmetry principle might cause their total net contribution to vanish. An immediate intuitive counterargument to this suggestion is the comment that no-one has ever seen a macroscopic body speed up as a result of friction! Thus a cancellation would be surprising in the light of our physical picture of the renormalisation group flow (of unitary theories) as the motion of a (supersymmetric) particle in coupling constant space, under the influence of an external magnetic field [20, 16]. Indeed, as we shall now explain, the unitarity of the truncated effective light-particle field theory guarantees that all quantum friction terms tend to increase the entropy, in accord with everyday experience, and hence cannot cancel in the path-integral sum.

In our case, it is easy to see that the renormalization group flow is irreversible, as has also been argued in ref. [13] on the basis of the isomorphism of the space-time foam theory of vortices and spikes with a Hall conductor. The rate of change of the Lagrange function $\Phi(6)$ is given by

$$
\dot{\Phi}=\beta^{i} \frac{\partial \Phi}{\partial q^{i}}
$$

which can be rewritten using equation (田)

$$
\dot{\Phi}=-\beta^{i} G_{i j} \beta^{j}
$$

Since the low-energy effective field theory of the light degrees of freedom should be unitary by itself, the metric $G^{i j}$ in the space of coupling constants must be positive definite [14]. Hence the Lagrange function decreases monotonically, corresponding 
to a monotonic change in the effective central charge. This in turn corresponds to a monotonic increase in the entropy, as can be seen explicitly from the expression (15) for the rate of change of the quantum entropy. The commutator factor in (14) can be rewritten as

$$
-i\left[\rho(H), q^{i}\right]=\frac{\partial \rho}{\partial p_{i}}=\frac{\partial \rho}{\partial H} \dot{q}^{i}=\frac{\partial \rho}{\partial H} \beta^{i}
$$

Substituting this expression into equation (15), we find

$$
\dot{S}=\operatorname{Tr} \beta^{i} G_{i j} \beta^{j} \frac{\partial \rho}{\partial H} \ln \rho
$$

In systems that exchange energy with their environment, as is our case, the density matrix is actually given by

$$
\rho=e^{\beta(F-H)}
$$

where $F$ is the free energy of the system. In the particular case of strings, $F$ is identified with the effective action $\Phi$ [15]. Thus, taking into account the facts that $\ln \rho<0$, and that for unitary $\sigma$-models $\beta^{i} G_{i j} \beta^{j}=2|z|^{4}<\Theta(z, \bar{z}), \Theta(0)>$ is positive definite [14], one observes that the quantum entropy does indeed increase monotonically thanks to the unitarity of the effective low-energy theory. This simple and general argument excludes the possibility of a sneaky cancellation between different contributions to the quantum friction.

We conclude this section by noting an essential difference between wormhole calculus and the type of quantum gravitational physics that we are discussing in this paper. Wormholes connect different parts of the target space-time via throats. Classical gauge symmetries that are carried by particles falling into one end of the wormhole are carried out by particles expelled at the other end, and there is no sign of information loss. Wormholes can give rise to non-local effects in space-time, which may be physically dubious for other reasons, but do not cause obvious problems for conventional quantum field theory or quantum mechanics. We do not address here the question whether string theory admits such wormholes. The defects on the world-sheet that we discuss here correspond to Minkowski space black holes without throats, where, as discussed above, information is transferred monotonically by $W$ symmetries to unobserved massive string modes, resulting in the above-mentioned monotonic increase in the apparent entropy of the observable light degrees of freedom.

\section{Simple Models of Quantum Friction Effects}

Before making an order-of-magnitude estimate of quantum friction effects in the low-energy effective field theory derived from string, we first remind the reader of the standard formalism for microscopic systems interacting with "environmental" oscillator modes. This enables us to make contact with the previous literature on 
the transition between the quantum behaviour of microscopic systems and the classical behaviour of macroscopic systems. The standard "environmental" formalism assumes weak perturbations around the equilibrium state of the microscopic system, which is justified in our case by the suppression of Planck-mass excitations at low energies. The effective lagrangian for a single harmonic oscillator coupled to "environmental" oscillators representing the infinite set of massive string modes is

$$
L=\frac{1}{2} M \dot{q}^{2}-V(q)+\frac{1}{2} \sum_{j}\left(m_{j} \dot{x}_{j}^{2}-m_{j} \omega_{j}^{2} x_{j}^{2}\right)-\sum_{j} F_{j}(q) x_{j}-\sum_{j} \frac{F_{j}(q)^{2}}{2 m_{j} \omega_{j}^{2}}
$$

where the last two terms represent the interactions. The density matrix of the system (29) can be expressed as a path integral in the usual way. The reduced density matrix describing the evolution of the primary microscopic harmonic oscillator from $q=q_{i}$ at time $t=0$ to $q=q_{f}$ at time $T$ is then given by

$$
K\left(q_{i}, q_{f}, T\right)=K_{0}(T) \int_{q(0)=q_{i}}^{q(T)=q_{f}} D q(\tau) e^{-S_{e f f}[q(\tau)] / \hbar}
$$

where the prefactor $K_{0}(T)$ involves only the environmental oscillator frequencies [21], and can be absorbed in the normalisation of the uncoupled case. The Euclidean effective action $S_{\text {eff }}$ is given by

$$
S_{e f f}[q(\tau)]=\int_{0}^{T} d \tau\left(\frac{1}{2} M \dot{q}^{2}+V(q)\right)+\frac{1}{2} \int_{-\infty}^{+\infty} d \tau^{\prime} \int_{0}^{T} d \tau \alpha\left(\tau-\tau^{\prime}\right)\left(q(\tau)-q\left(\tau^{\prime}\right)\right)^{2}
$$

where

$$
\alpha\left(\tau-\tau^{\prime}\right) \equiv \sum_{n} \frac{C_{n}^{2}}{4 m_{n} \omega_{n}} e^{-\omega_{n}\left(\tau-\tau^{\prime}\right)}
$$

In deriving these results, we have simplified matters by assuming linearity in the interactions: $F_{j}(q)=q C_{j}$, corresponding to a Wilson renormalisation scheme in string theory [16]. In this case it can be shown that $\alpha$ is given by the asymptotic form of the so-called Drude model [21]

$$
\alpha\left(\tau-\tau^{\prime}\right)=\eta \frac{1}{\left(\tau-\tau^{\prime}\right)^{2}}
$$

where $\eta$ is the "environmental" friction coefficient, given by

$$
\eta \equiv \sum_{j} \frac{C_{j}^{2}}{m_{j} \omega_{j}^{2}} \delta\left(\omega-\omega_{j}\right)
$$

We see from Eq. (32) that the strengths of the dissipation terms are suppressed for heavy "environmental" oscillators, a feature that we might expect to carry over to more massive modes in string theory. The "environmental" dissipation effects (32) are suppressed linearly in the large masses or frequencies. If this result is carried over to string theory (with the correspondence of the oscillator frequencies $\omega_{j}$ in (34) to the string mass levels), the magnitude of the stringy quantum friction effects could be comparable to the upper bounds established in refs. [3, 5] for the neutral kaon and neutron systems. 


\section{Order-of-Magnitude Estimate in String Theory}

In previous sections we have seen that in two-dimensional string theory the exactly marginal operators that turn on backgrounds for the light degrees of freedom in the presence of a black-hole contain necessarily massive string states which are discrete solitonic states, that are not measured in laboratory experiments. To estimate the orders of magnitude of the quantum- gravitational friction term in (14) and of the rate of change of the entropy (27), we need to discuss the magnitude of the renormalization functions $\beta^{i}$. In general, one has

$$
\beta^{i}=\sum_{N=1}^{\infty} C_{i_{1} \ldots i_{N}}^{i} g^{i_{1}} \ldots g^{i_{N}}
$$

where the coefficients $C_{i_{1} \ldots i_{N}}^{i}$ are not totally symmetric among covariant and contravariant indices. The usual on-shell string N-point amplitudes coincide with the expansion coefficients $\gamma_{i_{1} \ldots i_{N}}=<\int d^{2} z_{1} V_{i_{1}} \ldots \int d^{2} z_{N} V_{i_{N}}>$ of the covariant $\beta_{i} \equiv G_{i j} \beta^{j}$ [26, 27]. These coefficients are totally symmetric as follows from the flow equation (4). Factorization implies that all higher-order coefficients are obtainable from 3point functions [27]. If one chooses an appropriate Wilson renormalization scheme, all contact terms are eliminated from these 3-point functions if the boundaries of moduli space are treated correctly [28], as we assume for the coset black hole model, in which case the metric tensor $G^{i} j$ in coupling constant space is flat with its nonzero entries $O(1)$ ?.

For exactly marginal operators with both light- and heavy-state terms: $V_{i}=$ $V_{i}^{(L)}+V_{i}^{(H)}$, such as $L_{0}^{1} \bar{L}_{0}^{1}(17)$, we have

$$
0=\beta^{i}=G^{i j}\left(S_{0}\right) \sum_{N=1}^{\infty} g^{i_{1}} \ldots g^{i_{N}}<\Pi_{n=1}^{N}\left(V_{i_{n}}^{(L)}+V_{i_{n}}^{(H)}\right)>
$$

Therefore, defining the light-state renormalization coefficients

$$
\hat{\beta}^{i}=\sum_{N=1}^{\infty} G^{i j}\left(S_{0}\right) g^{i_{1}} \ldots g^{i_{N}}<\Pi_{n=1}^{N} V_{i_{n}}^{(L)}>
$$

we find

$$
\hat{\beta}^{i}=-\sum_{N=1}^{\infty} \sum_{M=1}^{N} g^{i_{1}} \ldots g^{i_{N}}<\Pi_{m=1}^{M} V_{i_{m}}^{(M)} \Pi_{n=1}^{N-M} V_{i_{n}}^{(L)}>
$$

\footnotetext{
3 The existence of such a Wilson scheme, and hence the vanishing of such contact terms, is subtle in certain cases such as supersymmetric strings [29], requiring a careful definition of correlation functions with colliding punctures [28. Thus it might not be easy for this scheme to be implemented in practice in the black hole case, whose singularity is associated with a twisted supersymmetric fixed point [30]. If a conventional renormalisation scheme were adopted in this case [31], it would give non-linear friction. However, we believe that physical observables would not be affected, since the corresponding conformal field theory is well-defined even at the singularity [23].
} 
The effective $\beta^{i}$ appearing in sections 2 and 3 are in fact the $\hat{\beta}^{i}$ (37) in the lightfield theory, and we now revert to the hatless notation of those earlier sections. As we have seen, these effective $\beta^{i}$ are non-vanishing to the extent that the mixed light-heavy N-point functions in (36) are non-zero.

To estimate these, we use the Euclidean counterpart of the $S L(2, R) / O(1,1) \mathrm{WZ}$ coset model [23], which can be mapped [32 into a $c=1$ matrix model with a modified cosmological constant on the world-sheet f: in their notation

$$
2 \pi \mu \beta \bar{\beta} \exp \left(-2 \frac{\phi}{\alpha_{+}}\right) \equiv \mu V_{1,1}^{(-)}
$$

where $\beta, \bar{\beta}$ are chiral bosons used along with the free field $\phi$ and another chiral boson $\gamma$ to parametrise $S L(2, R)$ 32]. The scale $\mu$ is related to the mass of the black hole by $\mu=M_{B H} / M_{\text {Planck }}$, and the massive string states are represented in general by

$$
V_{r_{1} r_{2}}^{( \pm)}=\left[\partial^{r_{1} r_{2}} X+\ldots\right] e^{i \frac{r_{1}-r_{2}}{\sqrt{2}} \pm \beta_{r_{1} r_{2}}^{ \pm} \phi}
$$

The (-) states are often discarded in usual Liouville theory [19], but play a key role here, as we shall see. In particular, following ref. [32], we have the following representation for the WZ model representing a two-dimensional black hole [23]

$$
S_{W Z}=S_{c=1, \mu=0}+\mu V_{1,1}^{(-)}
$$

The $(+)$ states are massive string modes, so the correlation functions of interest to us can be expressed as

$$
\sum_{l=0}^{+\infty} \frac{1}{l !} \mu^{l}<\Pi_{i=1}^{N^{\prime}} V_{r_{i} r_{i+1}}^{(+)} \Pi_{j=1}^{M} V_{j}^{0} \Pi_{m=1}^{l} V_{r_{m-1} r_{m}}^{(-)}>_{1}
$$

where the superscript (0) denotes a massless "tachyon" state, and the subscript 1 on the v.e.v. denotes the fact that it is calculated in a $c=1$ matrix model with $\mu=0$.

We now seek to understand which correlation functions are in general non-vanishing, and how they scale with $\mu$. The first important observation in this regard is that correlators of $V^{(-)}$operators alone vanish: only correlators with at least one $V^{(+)}$ operator are non-zero. This means that the black hole dynamics necessarily turns on physical massive string states, as we had seen previously from the point of view of the $W$-symmetry linking massless and massive modes [8, 12]. The second comment is that the non-zero correlators contain in general logarithmic factors that violate the expected power-law scaling with $\mu$. Specifically, there are factors of $(\ln \mu)^{ \pm 1}$ for

${ }^{4}$ Although Euclidean and Minkowski coset models are not physically equivalent in this formalism [11, 33], the Euclidean version will be sufficient for our order-of-magnitude estimates if we assume that the analytic continuation in target space needed to produce Minkowski black holes does not affect the scaling arguments we are using below. 
insertions of $V^{( \pm)}$operators respectively, associated with divergences close to the Fermi level and representing physical pole conditions in amplitudes [34, 35]. Thus we have the following general scaling laws:

$$
<\Pi_{i=1}^{2 N^{\prime}} V_{r_{i-1} r_{i}}^{(+)} \Pi_{j=1}^{M} V_{j}^{0} \Pi_{m=1}^{2 N} V_{r_{m-1} r_{m}}^{(-)}>_{1} \propto\left\{\begin{array}{c}
\mu^{s}(\ln \mu)^{N^{\prime}-N} \times F(\{k\}) \text { for } N^{\prime}-N>0 \\
0 \quad \text { otherwise }
\end{array}\right.
$$

where $F(\{k\})$ is a kinematical factor, and the power $s$ is determined by naive kinematical scaling considerations. It is related to the Liouville-energy non-conservation which arises from the reality of the coupling of the Liouville field in the expression for the vertex operators [36]. For resonant amplitudes, $s=0$. For general $s$ one can only compute the amplitudes by analytic continuation from the integer $s$ case [37. Such a procedure is assumed in the following.

We see in the general scaling law (43) logarithmic factors in the non-zero correlators that appear to vanish as $\ln \mu \rightarrow 0$, i.e., as $\mu \rightarrow 1$. Therefore we are led to conclude that the contributions to the quantum-gravitational friction are suppressed for configurations with $\mu \simeq 1$. Formally, the expression (43) is ill-defined for $\mu \rightarrow 0$, i.e., $\ln \mu \rightarrow-\infty$. This we interpret as a reflection of the well-known ill-definition of the $c=1$ matrix model itself when $\mu=0$. Analytic continuation is not safe in this regime, since if one formally evaluates the amplitudes in the limit $\mu \rightarrow 0$ by analytically continuing to $s=$ integer $>N^{\prime}-N$ in (43), then the amplitudes vanish. We interpret these subtleties as follows: as discussed in ref. [13], we believe that the appropriate string vacuum is a space-time foam consisting of a plasma of spikes on the world-sheet corresponding to microscopic Minkowski-space black holes. The end-state of black hole decay is a microscopic black hole that is indistinguishable from this foam, so the latter should be subtracted and serve as a regulator for black hole physics. This would therefore be dominated in our application by configurations with $\mu \geq 1$ in the gravitational path integral analogue of the simple model $(32)$.

Incorporating the estimate (43) into the expression (38) for the effective lightfield renormalization coefficients $\beta^{i}$, and then using the path integral $(20,21)$ to perform the sum over microscopic Minkowski black holes in the space-time foam, which is dominated by configurations with $\mu=M_{B H} / M_{\text {Planck }} \geq 1$ as discussed above, we estimate that the coefficients of the quantum-gravitational friction terms in (14,27) are $O(1)$ in Planck units. Thus their effects are suppressed by dimensional powers of $M_{\text {Planck }}$, and the largest could be $O\left(M_{\text {Planck }}^{-1}\right)$ as in the simple model (32). Their detailed evaluation requires more knowledge of the effective low-energy theory derived from string, and goes beyond the scope of this paper. 


\section{Collapse of the wave-function}

It has been argued [6] that the microscopic entropy increase offered by quantum gravity effects may cause the collapse of macroscopic wave functions and their transition from quantum to classical behaviour. We now discuss whether quantum quantum-gravitational friction (14) and the rate of entropy increase (27) could have this effect. We start from the simple model for friction described in section 4 . In

the string case, the renormalized $\sigma$-model couplings play the rôles of coordinates $q$, and $\tau$ is the renormalization group scale parameter, identified with target time. Since the $q$ 's are almost but not exactly marginal in the truncated effective lightmode field theory, the dominant contributions to the path-integral in the asymptotic Drude model (33) come from the limit where $\tau \rightarrow \tau^{\prime}$. In this case eq (31) may be written as

$$
\begin{array}{r}
\rho\left(q_{i}, q_{f}, T\right) / \rho_{S}\left(q_{i}, q_{f}, T\right) \simeq e^{-\eta \int_{0}^{T} d \tau \int_{\tau-\epsilon}^{\tau+\epsilon} d \tau^{\prime} \frac{\left(q(\tau)-q\left(\tau^{\prime}\right)\right)^{2}}{\left(\tau-\tau^{\prime}\right)^{2}}} \simeq \\
e^{-\eta \int_{0}^{T} d \tau \int_{\tau^{\prime} \simeq \tau} d \tau^{\prime} \beta^{i} G_{i j}\left(S_{0}\right) \beta^{j}} \simeq e^{-D T\left(\mathbf{q}_{\mathbf{i}}-\mathbf{q}_{\mathbf{f}}\right)^{2}+\ldots}
\end{array}
$$

where $D$ is a small constant, proportional to the sum of the squares of the effective anomalous dimensions of the renormalised couplings $q^{i}$. We reinterpret (31) as representing the overlap, after an elapsed time interval $T$, between quantum systems localized at different values $\mathbf{q}=\mathbf{q}_{\mathbf{i}}, \mathbf{q}_{\mathbf{f}}$ of the coordinates, and the subscript " $\mathrm{S}$ " denotes quantities evaluated in Schroedinger quantum mechanics. Equation (44) exhibits a quadratic dependence on the $\beta$-functions in this simple mechanical model for friction. It captures all the essential features of the complicated string case, in which the light-mode effective $\beta$-functions are suppressed by inverse powers of $M_{\text {Planck }}$, as we discussed in the previous section.

The vanishing of off-diagonal terms in the density matrix leads in general to the collapse of the wave-function. This effect is usually negligible for microscopic light-mode systems - except possibly for special cases such as the $K^{0}-\bar{K}^{0}$ system discussed elsewhere [3, 5] - but it is enhanced for macroscopic systems. This was first derived in ref. [6] in the context of a wormhole model, but the result is more general and applies here. We consider a macroscopic body containing many particles with coordinates $q^{i}, i=1,2, \ldots N$. Decomposing these into a centre-of-mass coordinate $Q$ and relative coordinates $\Delta q^{i},(i=1,2, \ldots N)$, writing the full density matrix as $\rho_{q}=\rho_{Q} \rho_{\Delta q}$, and assuming friction terms $-D t\left(q^{i}-q^{i}\right)^{2}(i=1,2, \ldots N)$, the equation for the centre-of-mass motion is

$$
\dot{\rho}_{Q} \simeq i\left[\rho_{Q}, H(Q)\right] \rho_{\Delta q}+i\left[\rho_{\Delta q}, H(\Delta q)\right] \rho_{Q}-\sum_{i=1}^{N} D\left(q^{i}-q^{i}\right)^{2} \rho\left(q^{i}, q^{i}\right)
$$

Tracing over the relative coordinates $\Delta q^{i}$, we find

$$
\dot{\rho}_{Q} \simeq i\left[\rho_{Q}, H(Q)\right]-N D\left(Q-Q^{\prime}\right)^{2} \rho_{Q}
$$


and hence

$$
\rho\left(Q^{\prime}, Q, t\right) / \rho_{S}\left(Q^{\prime}, Q, t\right) \simeq e^{-N D t\left(Q^{\prime}-Q\right)^{2}}
$$

where we see an enhanced suppression for bodies containing many particles. As an illustration, if $D \simeq m_{\text {proton }}^{6} / M_{\text {Planck }}^{3}$ as in ref. [6], then the locations of bodies with $N \geq 10^{24}$ constituent particles (cf Avogadro's number) are fixed to within a Bohr radius within about $10^{-7}$ sec. A similar mechanism for wave function collapse was introduced phenomenologically in ref. [7] without reference to any microscopic model.

A final comment we would like to make concerns other possible effects of the space-time foam, which could also be enhanced macroscopically. As we have argued in [13], the space-time foam is represented as a plasma of world-sheet spikes, and one should examine the motion of a single light string mode in this plasma which is not yet feasible. We notice, however, that taking into account the no-net-force condition among the spikes of the plasma [13], we can define a 'centre-of-mass' coordinate for the black hole moduli and examine the motion of the tachyon in a single coset model, representing in some sense this 'collective' effect. In such a case, following [6], one would have an enhanced macroscopic non-quantum mechanical effect if there were microscopic violations of the no-net-force condition, leading to a motion of the centre of mass of the black-hole moduli. Such effects are left for future investigations. However one should keep in mind that they might also lead to "observable" modifications of quantum mechanics.

\section{Discussion}

The following remarks may help the reader to understand intuitively the relation of this work to other studies. As we have emphasized previously [12], the scattering of light particles off a black hole resembles the Callan-Rubakov [38] description of the scattering of charged particles off a grand unified monopole. There, conservation laws enforce selection rules on the scattered particles, and here similar selection rules ensure that quantum coherence is conserved by the full light/heavy particle system. The situation we have discussed in this paper is more akin to the 't Hooft [39] analysis of instanton effects in the Standard Model. In that case, scattering light particles have a very small probability of "meeting" an instanton, but then it generates new physics. In our case, light particles again have a very small probability of "meeting" a microscopic or virtual black hole, but then it also generates new physics. Since the heaavy degrees of freedom are necessarily ignored in a light-particle scattering experiment, information and hence coherence are lost in this case.

We have laid out in this paper the conceptual basis for the modification of laboratory quantum mechanics in string theory. We re-emphasize that the full string theory is perfectly quantum-mechanical, but that experiments cannot see this unless they measure all the massive string states, which are linked to light states by 
an infinite set of $W$-symmetries. Observing these massive modes is an impossibility in realistic laboratory experiments. Therefore, one must work with an effective theory of the light degrees of freedom as an open system, with the corresponding modification (14) of the Liouville equation for the density matrix. A simple extension of this argument will lead to a non-factorizing $\$$-matrix description of the transition between initial and final density matrices in quantum field theory. The quantum-gravitational friction that we have identified in this paper appears able to explain qualitatively the transition from quantum-mechanical behaviour of microscopic systems to the classical behaviour of macroscopic systems containing Avogadro's number of elementary particles.

\section{Acknowledgements}

The work of D.V.N. is partially supported by DOE grant DE-FG05-91-ER-40633 and by a grant from Conoco Inc.

\section{References}

[1] M.B. Green, J.H. Schwarz and E. Witten, Superstrings Vol. I \& II (Cambridge University Press 1988).

[2] S. Hawking, Comm. Math. Phys. 43 (1975), 199; ibid 87 (1982), 395.

[3] J. Ellis, J.S. Hagelin, D.V. Nanopoulos and M. Srednicki, Nucl. Phys. B241 (1984), 381.

[4] Y.R. Shen, Phys. Rev. 155 (1967), 921;

A.S. Davydov and A. A. Serikov, Phys. Stat. Sol. B51 (1972), 57;

B.Ya. Zel'dovich, A.M. Perelomov, and V.S. Popov, Sov. Phys. JETP 28 (1969), 308 ;

For a recent review see : V. Gorini et al., Rep. Math. Phys. Vol. 13 (1978), 149.

[5] J. Ellis, N.E. Mavromatos and D.V. Nanopoulos, CERN and Texas A \& M Univ. preprint, CERN-TH.6596/92; CTP-TAMU-47/92; ACT-12/92 (following paper).

[6] J. Ellis, S. Mohanty and D.V. Nanopoulos, Phys. Lett. B221 (1989), 113; ibid B235 (1990), 305.

[7] G.C. Ghirardi, A. Rimini and T. Weber, Phys. Rev. D34 (1986), 470; G.C. Ghirardi, O. Nicrosini, A. Rimini and T. Weber, Nuov. Cim. 102B (1988), 383.

[8] J. Ellis, N.E. Mavromatos and D.V. Nanopoulos, Phys. Lett. B267 (1991), 465.

[9] J. Ellis, N.E. Mavromatos and D.V. Nanopoulos, Phys. Lett. B272 (1991), 261. 
[10] J. Ellis, N.E. Mavromatos and D.V. Nanopoulos, Phys. Lett. B278 (1992), 246.

[11] J. Ellis, N.E. Mavromatos and D.V. Nanopoulos, Phys. Lett. B276 (1992), 56.

[12] J. Ellis, N.E. Mavromatos and D.V. Nanopoulos, Phys. Lett. B284 (1992), 27, 43.

[13] J. Ellis, N.E. Mavromatos and D.V. Nanopoulos, CERN and Texas A \& M Univ. preprint, CERN-TH.6534/92; CTP-TAMU-47/92; ACT-12/92; CERNTH.6536/92; CTP-TAMU-48/92; ACT-13/92;

[14] A.B. Zamolodchikov, JETP Lett. 43 (1986), 730; Sov. J. Nucl. Phys. 46 (1987), 1090.

[15] N. Mavromatos and J.L. Miramontes, Phys. Lett. B212 (1988), 33; N. Mavromatos, Mod. Phys. Lett. A3 (1988), 1079; Phys. Rev. D39 (1989), 1659; A. Tseytlin, Phys. Lett. B194 (1987), 63; H. Osborn, Phys. Lett. B214 (1988), 555 .

[16] N.E. Mavromatos, J.L. Miramontes and J.M. Sanchez de Santos, Phys. Rev. D40 (1989), 535.

[17] I. Kogan, Univ. of British Columbia preprint UBCTP 91-13 (1991).

[18] I. Antoniadis, C. Bachas, J. Ellis and D.V. Nanopoulos, Phys. Lett. B211 (1988), 393; Nucl. Phys. B328 (1989), 117; Phys. Lett. (1991), 278.

[19] J. Polchinski, Nucl. Phys. B324 (1989), 123.

[20] C. Vafa, Phys. Lett. B212 (1988), 29; S. Das, G. Mandal and S. Wadia, Mod. Phys. Lett. A4 (1989), 745.

[21] R.P. Feynman and F.L. Vernon Jr., Ann. Phys. (NY) 94 (1963), 118;

A.O. Caldeira and A.J. Leggett, Ann. Phys. 149 (1983), 374.

[22] S. Chaudhuri and J. Lykken, FERMILAB preprint FERMI-PUB-92/169-T.

[23] E. Witten, Phys. Rev. D44 (1991), 314.

[24] G. Moore and N. Seiberg, Int. J. Mod. Phys. A7 (1992), 2601.

[25] I. Klebanov and A.M. Polyakov, Mod. Phys. Lett. A6 (1991), 3273;

A.M. Polyakov, Princeton Univ. preprint PUPT-1289 (1991).

[26] N.E. Mavromatos and J.L. Miramontes, Phys. Lett. B226 (1989), 291.

[27] R. Brustein, D. Nemeschansky and S. Yankielowicz, Nucl. Phys. B301 (1988), 224. 
[28] J. Distler and M. Doyle, Princeton Univ. preprint PUPT-1312 (1992).

[29] M.B. Green and N. Seiberg, Nucl. Phys. B299 (1988), 559.

[30] T. Eguchi, Mod. Phys. Lett. A7 (1992), 85.

[31] R. Dijkraaf, H. Verlinde and E. Verlinde, Nucl. Phys. B371 (1992), 269.

[32] M. Bershadsky and D. Kutasov, Phys. Lett. B266 (1991), 345.

[33] J. Distler and P. Nelson, Nucl. Phys. B374 (1992), 123.

[34] D. Gross, I. Klebanov and M.J. Newman, Nucl. Phys. B350 (1991), 621.

[35] P. di Francesco and D. Kutasov, Phys. Lett. B261 (1991), 385; Nucl. Phys. B375 (1992), 119.

[36] A.M. Polyakov, Mod. Phys. Lett. A6 (1991), 635.

[37] M. Goulian and M. Li, Phys. Rev. Lett. 66 (1991), 2051.

[38] C. Callan, Phys. Rev. D25 (1982), 2141;

V.A. Rubakov, Nucl. Phys. B203 (1982), 311;

V.A. Rubakov and M.S. Serebryakov, Nucl. Phys. B237 (1984),239.

[39] G. 't Hooft, Phys. Rev. Lett. 37 (1976), 8; Phys. Rev. D14 (1976), 3432; ibid D18 (1978), 2199. 